\title{
Balancing Sodium and Potassium: Estimates of Intake in a New Zealand Adult Population Sample
}

\author{
Rachael McLean 1,2,3, Julia Edmonds ${ }^{1}$, Sheila Williams ${ }^{2}$, Jim Mann ${ }^{1}$ and Sheila Skeaff 1,3 \\ 1 Department of Human Nutrition, University of Otago, P.O. Box 56, Dunedin 9054, New Zealand; \\ kiwijulia@yahoo.co.uk (J.E.); jim.mann@otago.ac.nz (J.M.); sheila.skeaff@otago.ac.nz (S.S.) \\ 2 Department of Preventive and Social Medicine, Dunedin School of Medicine, University of Otago, \\ P.O. Box 56, Dunedin 9054, New Zealand; sheila.williams@otago.ac.nz \\ 3 Nutrition Society of New Zealand, PO Box 2039, Whanganui 4543, New Zealand \\ * Correspondence: rachael.mclean@otago.ac.nz; Tel.: +64-3479-7943; Fax: +64-3479-7958
}

Received: 1 September 2015; Accepted: 22 October 2015; Published: 28 October 2015

\begin{abstract}
Dietary intakes of sodium and potassium are important determinants of blood pressure. We assessed sodium and potassium intake in a cross-sectional survey which included a random sample of New Zealand Adults aged 18 to 64 years from two New Zealand cities: Dunedin and Wellington. Participants completed a short questionnaire, had height, weight and blood pressure measured, and collected a $24 \mathrm{~h}$ urine sample. Mean $24 \mathrm{~h}$ sodium excretion was $3386 \mathrm{mg} /$ day $(95 \%$ CI 3221, 3551): $3865 \mathrm{mg} /$ day for men and for $2934 \mathrm{mg} /$ day women. Mean $24 \mathrm{~h}$ potassium excretion was $2738 \mathrm{mg} /$ day $(95 \%$ CI 2623, 2855): $3031 \mathrm{mg} /$ day for men and $2436 \mathrm{mg} /$ day for women. Mean sodium:potassium ratio was 2.2 (95\% CI 2.1, 2.3); 2.3 for men and 2.1 for women. Sodium intake was higher among younger people, men, those with a higher BMI and higher potassium excretion. Potassium excretion was higher among older people, men and those with a higher sodium excretion. New Zealand adults have high sodium intakes and low potassium intakes compared to recommended levels. This is likely to adversely affect population blood pressure levels as well as incidence of cardiovascular disease. A comprehensive public health programme to reduce dietary sodium intake and increase intake of fruit and vegetables is warranted.
\end{abstract}

Keywords: sodium; potassium; dietary; adult; New Zealand

\section{Introduction}

A dietary pattern which is low in sodium and high in fresh fruit and vegetables (and therefore high in potassium) has been recommended for blood pressure lowering, and is well supported by numerous randomised controlled trials [1-3]. The World Health Organization (WHO) 2013-2020 Global Action Plan for the Prevention and Control on Noncommunicable Diseases identifies nine voluntary global targets including a $25 \%$ reduction in risk of cardiovascular disease and raised blood pressure, and a 30\% reduction in mean population salt intake by 2025 [4]. WHO also recommends that adults consume a minimum of $400 \mathrm{~g}$ of fruit and vegetables per day (excluding starchy root vegetables such as potatoes) for the prevention of chronic disease [5]. A sodium: potassium intake ratio of approximately 1 has been recommended for optimal health [6,7], with higher ratios associated with elevated blood pressure and increased risk of cardiovascular disease [8]. WHO recommends a maximum sodium intake for adults of $2 \mathrm{~g}$ per day (equivalent to $5 \mathrm{~g}$ of salt) [7].

In New Zealand a number of previous population based surveys have shown that dietary potassium intakes are lower, and dietary sodium intakes are substantially above recommended levels. The most recent estimate of population potassium intake comes from $24 \mathrm{~h}$ diet recall used in the 2008/09 New Zealand Adult Nutrition Survey (2008/09NZANS). These results show the median 
usual daily intakes of potassium were $3449 \mathrm{mg} /$ day for men and $2757 \mathrm{mg} /$ day for women, with no significant differences by ethnicity or socio-economic status. These are below the recommended adequate intake (AI) [9]. The last reported data on sodium intakes in New Zealand are based on the collection of $24 \mathrm{~h}$ urine samples from healthy volunteers between 1993 and 1998 in two New Zealand provinces reporting a sodium intake of $3464 \mathrm{mg} /$ day (3854 for men and 3122 for women) [10]. We aimed to update an assessment of sodium and potassium intake from $24 \mathrm{~h}$ urine samples with biochemical validation of completeness (the gold standard [11]) in a random sample of adults living in two main cities in New Zealand.

\section{Experimental Section}

This cross-sectional survey was conducted between February and November 2012. Adults aged between 18 and 64 years were identified from the New Zealand electoral roll (including both the general and Māori rolls) in two cities in New Zealand (Wellington (population =191,000)) [12], and Dunedin (population $=119,000)$ ) [13]. A random sample with an equal number of men and women in each of three age groups (18-24 years, 25-44 years, and 45-64 years) was drawn from the electoral roll. Participants were invited by post, and followed up a maximum of three times using postcards and telephone contact if required, all participants have signed an informed consent form. Ethical approval was obtained from the University of Otago Ethics Committee (reference 11/244).

We anticipated proportions participating of 50\% in Dunedin and 35\% in Wellington based on results of a previous similar study [14]. Because the proportion participating among 18-24 year old men was low, a snowball (word of mouth) technique was used to recruit further participants who were not family members of existing participants in this demographic group. Participants were eligible if they met the following criteria: no history of thyroid, heart, or kidney disease; not taking any thyroid medication or had not started diuretics in the previous 2 weeks. They attended a clinic session with a trained interviewer and completed a questionnaire, which included sociodemographic information (age, sex, ethnicity, level of income), and medication use. Ethnicity was self-identified and participants were categorised into three ethnic groups: Māori, Pacific, and New Zealand European and Others (NZEO).

Participants underwent standardised height, weight and blood pressure measurements. Blood pressure was measured using the HEM-7221 (M6 comfort, Omron Healthcare, Inc., Lake Forest, IL, USA) automated sphygmomanometer with an appropriately sized cuff [15]. Three blood pressure measurements were taken one minute apart, and the mean of the second and third readings were used for this analysis.

Twenty-four hour urine collections were obtained following a protocol developed by the Pan American Health Organization Regional Expert Group for Cardiovascular Disease Prevention [16]. Participants were given collection equipment (including a two and five litre container, a jug and funnel and information sheet) and instructions for completing a $24 \mathrm{~h}$ urine collection on a day most convenient to them. They were asked to record time of collection (including start and finish time) and whether they had missed any urine collections during the $24 \mathrm{~h}$ period. After collection, participants were instructed to contact a courier company and asked to place equipment and completed $24 \mathrm{~h}$ urine sample in pre-paid courier bags which were subsequently couriered to local clinics. At the clinic, the total volume of each $24 \mathrm{~h}$ urine collection was recorded, and two aliquots stored at $-20^{\circ} \mathrm{C}$. Frozen urine samples from Wellington participants were couriered to Dunedin; all samples were stored at the Department of Human Nutrition, University of Otago, Dunedin at $-20{ }^{\circ} \mathrm{C}$ until analysis. Urine samples were thawed at room temperature, vortexed, and analysed in batches in Department of Human Nutrition laboratories. Urinary sodium and potassium were determined on a Roche Hitachi Cobas C311 ISE unit biochemical analyser using an ion selective electrode. Urinary creatinine was determined by Jaffe reaction using alkaline picrate (Roche Hitachi Cobas C311 analyser). Completeness of $24 \mathrm{~h}$ urine collection was assessed in three ways: firstly participants were asked to report whether they had missed any urine collections, and results were not included if they reported missing two or more collections. Secondly, $24 \mathrm{~h}$ creatinine excretion was estimated using a formula developed by Joossens and Geboers [17] (estimated $24 \mathrm{~h}$ creatinine 
excretion $(\mathrm{mg} /$ day $)=24 \times$ body weight $(\mathrm{kg})$ for men and $21 \times$ body weight for women $)$ with participants excluded with an observed $24 \mathrm{~h}$ creatinine excretion less than $60 \%$ of the estimated value [17]. Thirdly, collections were excluded if they were less than $0.5 \mathrm{~L}$ in volume.

\section{Statistical Analysis}

All statistical analyses were carried out using the statistical package STATA 11.1 (StataCorp LP. College Station, TX, USA). Both $24 \mathrm{~h}$ urinary excretion of sodium and potassium were used as estimates of daily dietary intake. Population means were estimated for men and women aged 18 to 64 years by weighting data for each respondent according to population estimates by age and sex from the New Zealand Census for 2012 [18]. The Stata survey command was used to estimate population means and confidence intervals [19]. Linear regression analysis was used to determine the effect of age, sex (male, female) and $24 \mathrm{~h}$ sodium excretion. As $24 \mathrm{~h}$ sodium excretion results are slightly positively skewed, the regression analysis was undertaken with both log-transformed and untransformed $24 \mathrm{~h}$ sodium excretion data.

\section{Results}

778 (57\%) of the 1525 invited did not respond to any postal invitations, three had died and 149 invitation letters were returned indicating incorrect address. Of the 598 who responded, 305 people declined to participate, and 16 were ineligible, leaving 274 who agreed to participate in the study. Of these only 251 completed the study- a final proportion participating of $17 \%$ of those initially invited. A further 54 people recruited via snowball sampling complemented the sample, with 50 completing the study. Of those who completed the study two $24 \mathrm{~h}$ urine samples were excluded because they were less than $0.5 \mathrm{~L}(0.42 \mathrm{~L}$ and $0.49 \mathrm{~L})$. No participants reported missing any collections, and none were excluded on the basis of inadequate creatinine excretion. The final sample included 299 participants.

Baseline characteristics of those included in the final analysis are presented in Table 1. 51\% were women and $94 \%$ were of NZEO ethnicity. Six percent of participants $(n=18)$ reported that they were taking medication for high blood pressure, and of these only two reported taking diuretic medications (both thiazide diuretics). Mean (SD) urine volume was $1.99 \mathrm{~L}(0.95)$ with a range of 0.57 to $7.34 \mathrm{~L} ; 2.08$ (1.06) L for men and $1.91(0.83) \mathrm{L}$ for women. Results from the urinary analysis for sodium, potassium and creatinine are shown in Table 2. Mean $24 \mathrm{~h}$ sodium excretion was 3386 $\mathrm{mg} /$ day $(95 \%$ CI 3221, 3551): $3865 \mathrm{mg} /$ day for men and $2934 \mathrm{mg} /$ day for women. Mean $24 \mathrm{~h}$ potassium excretion was $2738 \mathrm{mg} /$ day (95\% CI 2623, 2855): $3031 \mathrm{mg} /$ day for men and $2436 \mathrm{mg} /$ day for women. Excluding one outlier of 10,605 mg/day, the mean $24 \mathrm{~h}$ potassium excretion was 2712 $\mathrm{mg} /$ day $(95 \%$ CI 2608, 2816). Mean sodium:potassium ratio was 2.2 (95\% CI 2.1, 2.3); 2.3 for men and 2.1 for women. Means weighted to reflect population proportions by age and sex are: for sodium excretion $3377 \mathrm{mg} /$ day (95\% CI 3202, 3551), potassium excretion $2744 \mathrm{mg} /$ day $(95 \%$ CI 2632, 2855) and sodium:potassium ratio 2.2 (95\% CI 2.1, 2.3). Mean $24 \mathrm{~h}$ creatinine excretion was $1422 \mathrm{mg} /$ day: $1710 \mathrm{mg} /$ day for men and for $1151 \mathrm{mg} /$ day women. The correlation between $24 \mathrm{~h}$ sodium excretion and $24 \mathrm{~h}$ potassium excretion was $0.36(p<0.001)$

Table 1. Baseline Characteristics of Participants.

\begin{tabular}{cccc}
\hline Characteristics & Men $(\boldsymbol{N}=\mathbf{1 4 5})$ & Women $(\boldsymbol{N}=\mathbf{1 5 4})$ & Total $(\boldsymbol{N}=\mathbf{2 9 9})$ \\
\hline City of residence: (number $(\%))$ & & & \\
Dunedin & $75(52)$ & $83(54)$ & $158(53)$ \\
Wellington & $70(48)$ & $71(46)$ & $141(47)$ \\
Age (number $(\%))$ & & & \\
18-24 years & $44(30)$ & $41(26)$ & $85(28)$ \\
25-44 years & $49(34)$ & $55(36)$ & $104(35)$ \\
45-64 years & $52(36)$ & $58(38)$ & $110(37)$ \\
\hline
\end{tabular}




\begin{tabular}{cccc}
\hline Ethnicity: (number (\%)) & & & \\
\hline Māori & $3(2)$ & $9(6)$ & $12(4)$ \\
Pacific & $2(1)$ & $5(3)$ & $7(2)$ \\
NZEO & $140(97)$ & $140(91)$ & $280(94)$ \\
Income: (number (\%)) & & & \\
<50,000 NZD per year & $74(51)$ & $100(66)$ & $174(59)$ \\
$>50,000$ NZD per year & $62(43)$ & $36(24)$ & $98(33)$ \\
Did not respond & $9(6)$ & $15(10)$ & $24(8)$ \\
Body size: mean (sd) & & & \\
Height (cm) & $177.2(7.3)$ & $164.3(5.9)$ & $170.5(9.2)$ \\
Weight (kg) & $83.3(15.0)$ & $72.6(16.2)$ & $77.8(16.2)$ \\
BMI (kg/m²) & $26.5(4.4)$ & $26.9(5.8)$ & $26.7(5.2)$ \\
Blood pressure: mean (sd) & & & \\
Systolic (mmHg) & $13215)$ & $120(14)$ & $126(16)$ \\
Diastolic (mmHg) & $78(12)$ & $76(11)$ & $77(11)$ \\
\hline
\end{tabular}

NZEO: New Zealand European and Other; NZD: New Zealand Dollar; BMI: Body Mass Index.

Table 2. The $24 \mathrm{~h}$ sodium, potassium and creatinine excretion: mean, standard deviation (sd) and $95 \%$ Confidence Interval $(95 \% \mathrm{CI})$.

\begin{tabular}{|c|c|c|c|c|c|c|c|}
\hline \multirow[t]{2}{*}{ Group } & \multicolumn{2}{|c|}{ Sodium (mg/day) } & \multicolumn{2}{|c|}{ Potassium (mg/day) * } & \multicolumn{2}{|c|}{$\begin{array}{c}\text { Sodium:Potassium } \\
\text { Molar Ratio * }\end{array}$} & \multirow{2}{*}{$\begin{array}{c}\text { Creatinine } \\
\text { (mg/day) }\end{array}$} \\
\hline & Mean(sd) & $95 \% \mathrm{CI}$ & Mean(sd) & $95 \% \mathrm{CI}$ & Mean(sd) & $95 \% \mathrm{CI}$ & \\
\hline \multicolumn{8}{|l|}{ Men: } \\
\hline 18-24 years & $3866(1554)$ & 3405,4327 & $2766(813)$ & 2525,3008 & $2.5(1.1)$ & $2.2,2.9$ & $1706(431)$ \\
\hline $25-44$ years & 3795 (1503) & 3372,7217 & 3058 (957) & 2786,3329 & $2.2(0.8)$ & $2.0,2.4$ & $1703(482)$ \\
\hline 45-64 years & $3931(1520)$ & 3517,4347 & $3085(1050)$ & 2798,3371 & $2.3(1.0)$ & $2.1,2.6$ & $1719(401)$ \\
\hline Total men & 3865 (1515) & 3618,4113 & 2979 (955) & 2822,3135 & $2.4(1.0)$ & $2.2,2.5$ & $1710(436)$ \\
\hline Weighted mean ${ }^{+}$ & 3854 & 3597,4111 & 3005 & 2837,3174 & 2.3 & $2.2,2.4$ & $\mathrm{~N} / \mathrm{A}$ \\
\hline \multicolumn{8}{|l|}{ Women: } \\
\hline 18-24 years & 3017 (954) & 2724,3311 & $2193(632)$ & 1999,2387 & $2.4(0.8)$ & $2.2,2.7$ & $1174(232)$ \\
\hline 25-44 years & 3035 (1241) & 2706,3365 & 2361 (839) & 2138,2583 & $2.3(0.9)$ & $2.1,2.6$ & $1188(283)$ \\
\hline 45-64 years & $2780(1401)$ & 2418,3142 & $2751(784)$ & 2549,2954 & $1.8(0.7)$ & $1.6,2.0$ & $1100(350)$ \\
\hline Total women & $2934(1235)$ & 2738,3130 & $2463(798)$ & 2337,2590 & $2.1(0.86)$ & $2.0,2.3$ & $1151(299)$ \\
\hline Weighted mean ${ }^{+}$ & 2926 & 2716,3137 & 2497 & 2363,2631 & 2.1 & $2.0,2.2$ & N/A \\
\hline Total men and women & $3386(1452)$ & 3221,3551 & $2712(913)$ & 2608,2816 & $2.2(0.93)$ & $2.1,2.3$ & $1422(465)$ \\
\hline
\end{tabular}

${ }^{*}$ excluding one outlier with potassium excretion of $10,605 \mathrm{mg} / \mathrm{day} ;{ }^{+}$weighted to reflect age and sex structure of the New Zealand population aged 18-64 years in 2012.

The majority $(77 \%, n=229))$ of participants had a sodium excretion that exceeded the Upper Level of Intake of $2300 \mathrm{mg} /$ day ( $85 \%$ of men and $69 \%$ of women). Only $23 \%(n=69)$ of participants had an adequate intake of potassium ( $2800 \mathrm{mg} /$ day for women and $3800 \mathrm{mg} /$ day for men) $(18 \%$ of men and $28 \%$ of women) according to current New Zealand Nutrient Reference Values [20].

Table 3 shows the results of multiple linear regression with $24 \mathrm{~h}$ sodium and $24 \mathrm{~h}$ potassium as outcome variables. In multiple linear regression, $24 \mathrm{~h}$ sodium excretion was significantly negatively associated with increasing age, and was positively associated with male sex, body weight and potassium excretion. There was no significant association between $24 \mathrm{~h}$ sodium excretion and systolic blood pressure when controlling for age, sex, body weight and $24 \mathrm{~h}$ potassium excretion. As $24 \mathrm{~h}$ sodium excretion results are positively skewed, the multivariate analysis was repeated using log-transformed $24 \mathrm{~h}$ sodium excretion data (results not shown). As this did not substantially change the substance of the results, those using un-transformed data are shown here. 
Table 3. Variables associated with $24 \mathrm{~h}$ urinary sodium and potassium excretion (multiple linear regression analyses) *.

\begin{tabular}{cccc}
\hline Models & $\boldsymbol{\beta}$ Coefficient & 95\% CI & $p$ \\
\hline Association with 24 h sodium excretion (mg/day) \\
\hline Age & -16 & $-27,-6$ & 0.003 \\
Male sex & 371 & 702,39 & $<0.028$ \\
Body weight (kg) & 23 & 13,32 & $<0.001$ \\
Potassium excretion (mg/day) & 0.53 & $0.36,0.69$ & $<0.001$ \\
Systolic Blood Pressure & 2 & $-8,13$ & 0.663 \\
\hline Association with 24 h potassium excretion (mg/day) \\
\hline Age & 11 & 4,17 & 0.002 \\
Male sex & 279 & 65,494 & 0.011 \\
Body weight (kg) & 3 & $-3,9$ & 0.369 \\
Sodium excretion (mg/day) & 0.22 & $0.15,0.29$ & $<0.001$ \\
Systolic Blood Pressure & 1 & $-6,8$ & 0.768 \\
\hline * excluding one outlier with potassium excretion of 10,605 mg/day.
\end{tabular}

Potassium excretion was positively associated with age and sodium excretion, and male sex. There was no significant association between $24 \mathrm{~h}$ potassium excretion and systolic blood pressure, when controlling for age, sex, body weight and $24 \mathrm{~h}$ sodium excretion.

\section{Discussion}

We found that this sample of New Zealand adults aged 18-64 had low potassium intakes and high sodium intakes compared with current New Zealand and international guidelines. This is reflected in the unfavourable sodium:potassium ratio which is above 1.0 for all groups. Sodium intake was higher among younger people, men, those with a higher BMI and higher potassium excretion. Potassium excretion was higher among older people, men and those with a higher sodium excretion. No association was demonstrated between sodium or potassium excretion and systolic blood pressure. These results indicate that improvements can be made to the dietary intake of New Zealand adults in order to reduce risk of cardiovascular disease, a leading cause of morbidity and mortality.

Our results also showed inadequate potassium intakes overall, and sodium:potassium ratios above 1.0 in all groups. This is consistent with potassium intake estimates from the 2008/09NZANS, which showed inadequate intakes from $24 \mathrm{~h}$ diet recall, (median usual daily intake of potassium of $3449 \mathrm{mg}$ for males and $2757 \mathrm{mg}$ for females, below the AI of $3800 \mathrm{mg} /$ day for men and $2800 \mathrm{mg} / \mathrm{day}$ for women). The $2008 / 09$ NZANS results show that only $59 \%$ of males and $72 \%$ of females reported eating three or more servings of vegetables a day, and only $55 \%$ of males and $66 \%$ of females reported eating two or more servings of fruit each day [9], which is likely to have contributed to the low observed potassium intakes in the 2008/09 NZANS as well as in our study.

Young adults, men and those with a higher BMI were more likely to have a high sodium intake. Sodium intake is highly correlated with energy intake [21] as it is highly prevalent in most processed foods, and this may be the main reason for these associations. Furthermore, young adults may be more likely to consume sodium dense foods such as takeaways and highly processed snacks than older adults [22]. In this survey young adults had a lower potassium intake suggesting a lower intake of fruit and vegetables than older adults. However, overall there was a positive correlation $(r=0.36)$ between sodium and potassium intake, and a positive association demonstrated on regression analysis, indicating that those with a higher sodium intake also had a higher potassium intake. This is likely to reflect an association with both sodium and potassium and energy intake, although this could not be assessed in this study. The lack of association demonstrated between systolic blood pressure and either sodium or potassium intake is not 
surprising, as this was a cross-sectional survey and not designed or powered to investigate this relationship.

Subject to the limitations inherent in studies of this type, the results of this survey suggest that New Zealand adults' sodium intake has remained remarkably stable since the late 1970s, despite decades of increasing evidence of the harmful effects of elevated sodium reduction. A 1975 survey of 1200 adults in a small South Island town (the Milton Survey) using $24 \mathrm{~h}$ urine collections, showed the average sodium excretion for men was $3979 \mathrm{mg} /$ day and for women was $3197 \mathrm{mg} /$ day [23]. A subsequent $24 \mathrm{~h}$ urinary assessment in two New Zealand provinces between 1993 and 1998 showed a mean sodium excretion of $3854 \mathrm{mg} /$ day for men and $3122 \mathrm{mg} /$ day for women [10]. Although it is disappointing that sodium intakes appear not to have decreased over this time, this pattern is consistent with estimates of sodium intakes in the United States and other countries over a similar time period $[24,25]$. It is also unsurprising for a number of reasons. Sodium is ubiquitous in the food supply. Estimates show that only about $10 \%$ of sodium intake from a Western-style diet is from salt added in the home in cooking or at the table, with around three quarters coming from sodium present in processed foods [26]. There is limited ability for individuals to control their own sodium intake in the absence of a concerted public health programme of reformulation of processed foods, especially as they find it difficult to interpret nutrition labels in this context [27]. Although some efforts are being made to lower population sodium intake, New Zealand has not implemented a comprehensive government led strategy such as that recently modelled in the United Kingdom. In New Zealand sodium reduction initiatives are currently led by the Heart Foundation in a programme called HeartSAFE: an industry led initiative to reduce the sodium content in bread, breakfast cereals, processed meat, savoury pies, soups, cheese, savoury snacks, cooking sauces and edible oil spreads [28]. This falls short of initiatives in other countries, such as South Africa (where a series of mandatory sodium concentration targets have recently been introduced for a range of processed foods) [29], and in the United Kingdom. The United Kingdom salt reduction programme was initiated in 2003 by the Food Standards Agency, and has included a consumer education programme, improvements in food labelling, as well as collaboration with food industry partners to achieve sodium reduction across a wide range of food products based on specific sodium concentration targets [30]. The implementation of the United Kingdom programme has been associated with a significant reduction in population sodium intake of $15 \%$ : from around 3800 $\mathrm{mg} /$ day in 2003 to around $3200 \mathrm{mg} /$ day in 2011. This estimate is based on $24 \mathrm{~h}$ urinary assessment in a population sample with a similar age range to that of our study. The reduction in sodium intake has been accompanied by a reduction in population blood pressure levels as well as reductions in mortality from ischaemic heart disease and stroke [30,31].

\section{Strengths and Limitations}

This is the first New Zealand survey estimating both sodium and potassium intakes using the gold standard $24 \mathrm{~h}$ urinary assessment in a randomly selected sample. A number of limitations exist however. Firstly, while we used the electoral roll to select a population sample, we only selected people from two cities: Wellington and Dunedin, which may not be generalisable to the whole New Zealand population. The proportion participating was relatively low at only $17 \%$, which may have biased results, however it is difficult to estimate how such a bias may have operated. There is some limited evidence that sodium intakes do not appear to be strongly associated with propensities to take part in surveys. In Australia, for example, a population based survey using $24 \mathrm{~h}$ urine collection had only $16 \%$ participating, although results were not significantly different between those invited participants and healthy volunteer recruits with respect to sodium excretion [32]. There was also no significant difference between participants recruited via the electoral roll, and those who volunteered through snowball sampling in our study. Compared with 2006 New Zealand Census data for each city $[12,13]$, there was a higher proportion of participants of NZEO ethnicity and with incomes greater than $\$ 50,000$ than the general population. It is possible therefore that based on income and the low proportion participating, those who agreed to participate were more likely to consume a more healthy diet (higher in potassium and lower in sodium) overall. Participants with a 
prior diagnosis of hypertension $(n=18)$ may be more likely to have been advised to restrict sodium intake, however hypertension is a common condition and the inclusion of these people is reflective of the population in this age range. Although 24-h urine is the gold-standard method of sodium and potassium intake, this is likely to be an under-estimate of total intake since $90 \%$ of sodium consumed is excreted in the urine. Furthermore, both under-and over collection of $24 \mathrm{~h}$ urine have been reported in other surveys [33]. While we used several widely accepted methods to exclude samples that were inadequate, under-collection of urine over the $24 \mathrm{~h}$ period cannot be excluded. Two participants reported being on long-term thiazide diuretics, however we believe that this is unlikely to have substantially altered sodium and potassium excretion results in this study.

\section{Conclusions}

New Zealand adults have high sodium intakes and low potassium intakes compared to recommended levels. This is likely to adversely affect population blood pressure levels as well as incidence of ischaemic heart disease and stroke. If New Zealand is to comply with the WHO recommendation to reduce sodium intake by $30 \%$ by 2025 , a comprehensive public health programme to reduce dietary sodium intake and increase intake of fruit and vegetables, thereby increasing potassium intake, is warranted.

Acknowledgments: We would like to thank the Ministry for Primary Industries for funding the study. We would like to thank the following people for assisting with subject recruitment: Emily Lonsdale-Cooper, Samantha Martin, and David Wolf. We would also like to thank Ashley Duncan and Michelle Harper for processing and analyzing urine samples.

Author Contributions: R.M.: primary author, statistical analysis, study design; aspects of study administration, participant recruitment, data collection and interpretation. J.E.: study administration, participant recruitment, data collection, and review of manuscript. S.W.: study design, statistical input, and random sampling from the New Zealand Electoral Roll, and review of manuscript. J.M.: study design, interpretation and review of manuscript. S.S.: study design; ethics; supervised overall study administration, participant recruitment, data collection and interpretation and analytical testing, and review of manuscript.

Conflicts of Interest: The authors declare no conflict of interest. This study was funded by the Ministry for Primary Industries (MPI) of the New Zealand Government. Whilst guidance was provided on the study design, MPI had no involvement in the collection, analyses, or interpretation of data; in the writing of the manuscript, and in the decision to publish the results.

\section{References}

1. Whelton, P.K.; He, J.; Cutler, J.A.; Brancati, F.L.; Appel, L.J;; Follmann, D.; Klag, M.J. Effects of Oral Potassium on Blood Pressure: Meta-analysis of Randomized Controlled Clinical Trials. JAMA J. Am. Med. Assoc. 1997, 277, 1624-1632.

2. He, F.; Li, J.; Macgregor, G. Effect of longer term modest salt reduction on blood pressure: Cochrane systematic review and meta-analysis of randomised trials. BMJ 2013, 346, 3.

3. Sacks, F.M.; Svetkey, L.P.; Vollmer, W.M.; Appel, L.J; Bray, G.A.; Harsha, D.; Obarzanek, E.; Conlin, P.R.; Miller, E.R., III; Simons-Morton, D.G.; et al. Effects on Blood Pressure of Reduced Dietary Sodium and the Dietary Approaches to Stop Hypertension (DASH) Diet. N. Engl. J. Med. 2001, 344, 3-10.

4. World Health Organization. Global Action Plan for the Prevention and Control of Noncommunicable Diseases 2013-2020; World Health Organization: Geneva, Switzerland, 2013.

5. World Health Organization. Global Strategy on Diet, Physical Activity and Health: Population Sodium Reduction Strategies. Available online: http://www.who.int/dietphysicalactivity/reducingsalt/en/ (accessed on 12 December 2013)

6. World Health Organization. Diet, Nutrition and the Prevention of Chronic Diseases: Report of a Joint WHO/FAO Expert Consultation; WHO Technical Report Series 916; World Health Organization: Geneva, Switzerland, 2003.

7. World Health Organization. Guideline: Sodium Intake for Adults and Children; World Health Organization: Geneva, Switzerland, 2012. 
8. Cook, N.R., Obarzanek, E.; Cutler, J.A.; Buring, J.E.; Rexrode, K.M.; Kumanyika, S.K.; Appel, L.J.; Whelton, P.K.; Trials of Hypertension Prevention Collaborative Research Group. Joint effects of sodium and potassium intake on subsequent cardiovascular disease: The Trials of Hypertension Prevention (TOHP) follow-up study. Arch. Intern. Med. 2009, 169, 32-40.

9. University of Otago and Ministry of Health. A Focus on Nutrition: Key Findings of the 2008/09 New Zealand Adult Nutrition Survey; Ministry of Health: Wellington, New Zealand, 2011.

10. Thomson, C.D.; Colls, A.J. Twenty-Four Hour Urinary Sodium Excretion in Seven Hundred Residents of Otago and Waikato; University of Otago: Dunedin, New Zealand, 1998.

11. Sanchez-Castillo, C.P., Branch, W.J.; James, W.P.T. A test of the validity of the lithium-marker technique for monitoring dietary sources of salt in man. Clin. Sci. 1987, 72, 87-94.

12. Statistics New Zealand. Census QuickStats about a Place: Wellington City. Available online: http://www.stats.govt.nz/Census/2013-census/profile-and-summary-reports/quickstats-about-a-place.aspx? request_value $=14438 \&$ tabname $=($ accessed on 14 December 2014$)$.

13. Statistics New Zealand. Census Quickstats about a Place: Dunedin City. Available online: http://www.stats.govt.nz/Census/2013-census/profile-and-summary-reports/quickstats-about-a-place.aspx ?request_value=15022\&tabname=Populationanddwellings (accessed on 14 December 2014)

14. Bradbury, K.E.; Skeaff, C.M.; Oey, I.; Williams, S.; Mann, J. Monitoring Voluntary Fortification of Bread with Folic Acid; Ministry of Agriculture and Forestry: Wellington, New Zealand, 2011.

15. Coleman, A.; Steel, S.; Freeman, P.; de Greeff, A.; Shennan, A. Validation of the Omron M7 (HEM-780-E) oscillometric blood pressure monitoring device according to the British Hypertension Society protocol. Blood Press. Monit. 2008, 13, 49-54.

16. WHO/PAHO. Regional Expert Group for Cardiovasular Disease Prevention through Population-wide Dietary Salt Reduction, Protocol for Population Level Sodium Determination in 24-hour Urine Samples; World Health Organization: Geneva, Switzerland, 2010.

17. Wang, C.-Y.; Cogswell, M.E.; Loria, C.M.; Chen, T.C.; Pfeiffer, C.M.; Swanson, C.A.; Caldwell, K.L.; Perrine, C.G.; Carriquiry, A.L.; Liu, K.; et al. Urinary Excretion of Sodium, Potassium, and Chloride, but not Iodine, Varies by Timing of Collection in a 24-Hour Calibration Study. J. Nutr. 2013, 143, 1276-1282.

18. Statistics New Zealand. Infoshare. Available online: http://www.stats.govt.nz/infoshare/Default.aspx (accessed on 12 May 2015).

19. StataCorp LP. Stata Data Analysis and Statistical Software Texas: StataCorp LP, 2012. Available online: http://www.stata.com/ (accessed on 28 April 2015).

20. Australian Department of Health and Ageing; National Health and Medical Research Council; New Zealand Ministry of Health. Nutrient Reference Values for Australia and New Zealand; Australian Department of Health and Ageing \& New Zealand Ministry of Health: Canberra, Australia, 2006.

21. Whelton, P.K.; Appel, L.J.; Sacco, R.L.; Anderson, C.A.M.; Antman, E.M.; Campbell, N.; Dunbar, S.B.; Frohlich, E.D.; Hall, J.E.; Jessup, M., et al. Sodium, Blood Pressure, and Cardiovascular Disease. Circulation 2012, 126, 2880-2889.

22. Smith, C.; Gray, A.R.; Fleming, E.A.; Parnell, W.R. Characteristics of fast-food/takeaway-food and restaurant/cafe-food consumers among New Zealand adults. Public Health Nutr. 2014, 17, 2368-2377.

23. Simpson, F.O., Nye, E.R.; Bolli, P.; Waal-Manning, H.J.; Goulding, A.W.; Phelan, E.L.; de Hamel, F.A.; Stewart, R.D.; Spears, G.F.; Leek, G.M.; et al. The Milton survey: Part 1, General methods, height, weight and 24-hour excretion of sodium, potassium, calcium, magnesium and creatinine. N. Z. Med. J. 1978, 87, 379-382.

24. Bernstein, A.M.; Willett, W.C. Trends in 24-h urinary sodium excretion in the United States, 1957-2003: A systematic review. Am. J. Clin. Nutr. 2010, 92, 1172-1180.

25. McCarron, D.A.; Kazaks, A.G.; Geerling, J.C.; Stern, J.S.; Graudal, N.A. Normal Range of Human Dietary Sodium Intake: A Perspective Based on 24-Hour Urinary Sodium Excretion Worldwide. Am. J. Hypertens. 2013, 26, 1218-1223.

26. Mattes, R.D.; Donnelly, D. Relative Contributions of Dietary Sodium Sources. J. Am. Coll. Nutr. 1991, 10, 383-393.

27. McLean, R.; Hoek, J.; Hedderley, D. Effects of alternative label formats on choice of high- and low-sodium products in a New Zealand population sample. Public Health Nutr. 2012, 15, 783-791. 
28. Heart Foundation of New Zealand. Sodium Targets. Available online: http://www.heartfoundation. org.nz/programmes-resources/food-industry-and-hospitality/heartsafe/industry-commitment-to-sodium-re duction (accessed on 18 December 2014).

29. World Health Organization Regional Office for Africa. Intersectoral Case Study: Stuccessful Sodium Regulation in South Africa; World Health Organization Regional Office for Africa: Johannesberg, Germany, 2013.

30. He, F.J., Brinsden, H.C.; MacGregor, G.A. Salt reduction in the United Kingdom: A successful experiment in public health. J. Hum. Hypertens. 2014, 28, 345-352.

31. He, F.J., Pombo-Rodrigues, S.; MacGregor, G.A. Salt reduction in England from 2003 to 2011: Its relationship to blood pressure, stroke and ischaemic heart disease mortality. BMJ Open 2014, 4, e004549.

32. Land, M.-A.; Webster, J.; Christoforou, A.; Praveen, D.; Jeffery, P.; Chalmers, J.; Smith, W.; Woodward, M.; Barzi, F.; Nowson, C.; et al. Salt intake assessed by $24 \mathrm{~h}$ urinary sodium excretion in a random and opportunistic sample in Australia. BMJ Open 2014, 4, e003720.

33. Joint Health Surveys Unit. A Survey of 24 Hour and Spot Urinary Sodium and Potassium Excretion in a Representative Sample of the Scottish Population; National Centre for Social Research: London, UK, 2007.

(C) 2015 by the authors; licensee MDPI, Basel, Switzerland. This article is an open access article distributed under the terms and conditions of the Creative Commons by Attribution (CC-BY) license (http://creativecommons.org/licenses/by/4.0/). 